\title{
A Framework for Integrating Change Management with Building Information Modeling
}

\author{
Fangxiao Liu ${ }^{1}$, Abdou Karim Jallow ${ }^{2}$, Chimay J. Anumba ${ }^{3}$ and Dinghao $\mathrm{Wu}^{4}$
}

${ }^{1}$ Department of Architectural Engineering, The Pennsylvania State University, University Park, PA 16802, USA; email: fzl116@psu.edu

${ }^{2}$ Department of Supply Chain and Information Systems, The Pennsylvania State University, University Park, PA 16802, USA; email: akj10@psu.edu

${ }^{3}$ Department of Architectural Engineering, The Pennsylvania State University, University Park, PA 16802, USA; email: anumba@psu.edu

${ }^{4}$ College of Information Sciences and Technology, The Pennsylvania State University, University Park, PA 16802, USA; email: dinghao@psu.edu

\section{ABSTRACT}

Managing changes is critical for a successful energy efficient retrofit project. Good change management (CM) enables the evaluation of change dependencies and effects, and it is essential to controlling and mitigating failure factors in projects. For example, inappropriate management may generate disruptive impacts on energy efficiency goals. The use of Building Information Modeling (BIM) has facilitated the delivery of both new build and retrofit projects but there is inadequate support for the management of changes, and very little research has been conducted to integrate CM processes within BIM. This paper aims to specify an integrated framework for embedding CM in BIM. The framework has been developed within the BIM Data Hub project, which focuses on developing a central repository of building information, to manage potential changes to IFC-based models. Current work on the BIM Data Hub focuses on retrieving domain-specific information from IFC models to conduct energy analysis. The outcomes of the analysis may result in recommendations for better energy performance, which would trigger potential changes. Such changes should be adequately managed across the building lifecycle. A comprehensive review of literature was conducted, including the developments and current status of CM and BIM adoption in energy efficient retrofits. The requirements and challenges for the integration of CM and BIM Data Hub were also investigated. Subsequently, the paper formulated a mechanism for how changes resulting from workflow execution can be updated on BIM models, and how change information can be disseminated among team members. A system platform was developed based on the integrated framework. Finally, the contributions and recommendations for future research are presented. 


\section{INTRODUCTION}

Changes occur in all phases of a construction project, and it is essential to implement an appropriate change management (CM) regime to reduce change-associated cost and time delays. Poor management of changes will lead to cost overruns and failure to meet the owners' goals and expectations (Jallow et al., 2013). For energy efficient retrofit projects, it is typically more complex than expected to mitigate the negative impacts of changes. Changes can be triggered by multi-discipline interactions, various requirements from different stakeholders, and multi-scale simulations and iterated calculations, etc. Besides, due to the constraints of time, space, information, and working environment in retrofits (Sanvido and Riggs, 1993), decisions are often made based on assumptions and experience, and it could cause changes to impact energy efficient goals.

Information management is a critical component of construction project management. Building Information Modeling (BIM) has emerged to facilitate efficient information exchange and sharing among different disciplines in an interactive environment (Eastman et al., 2011; Liu et al., 2013a), and it has been well developed and widely implemented in the architecture, engineering, and construction (AEC) industry (Taylor and Bernstein, 2009). The Building Information Modelserver (BIMserver) project, which is aimed to create a center of information for construction projects using IFC standard, developed an open source BIMserver platform to help users create their own BIMserver (BiMserver.org). However, the current approach to BIM has not been fully developed to support adequate management of changes (Langroodi and Staub-French, 2012), and little known research has been conducted to automate the process of updating change information in BIM models. This paper presents a framework to integrate BIM with the integrated change and knowledge management (CKM) approach (Liu et al., 2013b), and the focus is on the change management component.

\section{RELATED WORK}

Change management is an integral process to identify existing and already occurred changes, predict potential changes and prevent their impacts, and coordinate changes throughout the entire project (Voropajev, 1998). It includes the evaluation of change impacts, identification of change sources, and implementation of dependency control (Motawa et al., 2007). If changes are not properly managed, they can cause direct and indirect consequences (Sun et al., 2004). The major concern for direct effects is the cost of rework and time overrun, which is generated from uncertainties and poor information communication (Love et al., 1999). This is a waste of both time and money in construction projects, and it causes difficulties for decision-making (Hao et al., 2008). Indirect effects refer to the impacts that are not closely associated with a specific construction process and may have adverse effects on future plans. They can lead to the need to communicate with other project members, cash flow changes, and loss of 'float' (Sun et al., 2004). Good change management enables the evaluation of change dependencies and their associated effects, as well as the capture and dissemination of change history. 
In energy efficient retrofits, lack of information and working space can cause potential changes in the construction process. Any changes or errors can delay the whole project schedule, which conflicts with the proposed short construction time. In addition, the parties involved in a retrofit project not only need to collaborate with other trades, but also have to coordinate with original design drawings and specifications of the building (if available). Moreover, change and dependency problems that occur in a retrofit project often involve more participants and sources. Therefore, a well-planned change management strategy is indispensable and should be developed prior to the retrofit work.

This research was conducted as part of the BIM Data Hub project, which focuses on developing a central repository of building information and explores the possibility to integrate CKM into BIM by using the BIMserver platform. Current work in BIM Data Hub includes the retrieval of domain specific information (i.e., the exchange requirements specified in MVD) from IFC models for energy analysis (Jiang et al., 2012; Yu et al., 2013). This retrieved information can be used, for example an engineer (e.g. lighting engineer, mechanical engineer, etc.) to carry out simulations. In this process, changes may be recommended for better energy performance, and such changes should be collated adequately and updated on BIM models.

\section{THE INTEGRATED FRAMEWORK}

The CKM approach was developed to enhance the performance of project management work and facilitate energy efficient building retrofits. The integration of $\mathrm{CM}$ and BIM can be achieved by adopting the change management aspect of the CKM approach to enable information update of an IFC model.

Figure 1 illustrates the communication between the integrated CKM process and the BIM Data Hub. New change requests will be captured and managed in the CKM process, which includes change evaluation and approval, dependency checking, and knowledge capture and reuse, etc. When a change is approved, the information will be sent back to BIM Data Hub and updated on IFC models.

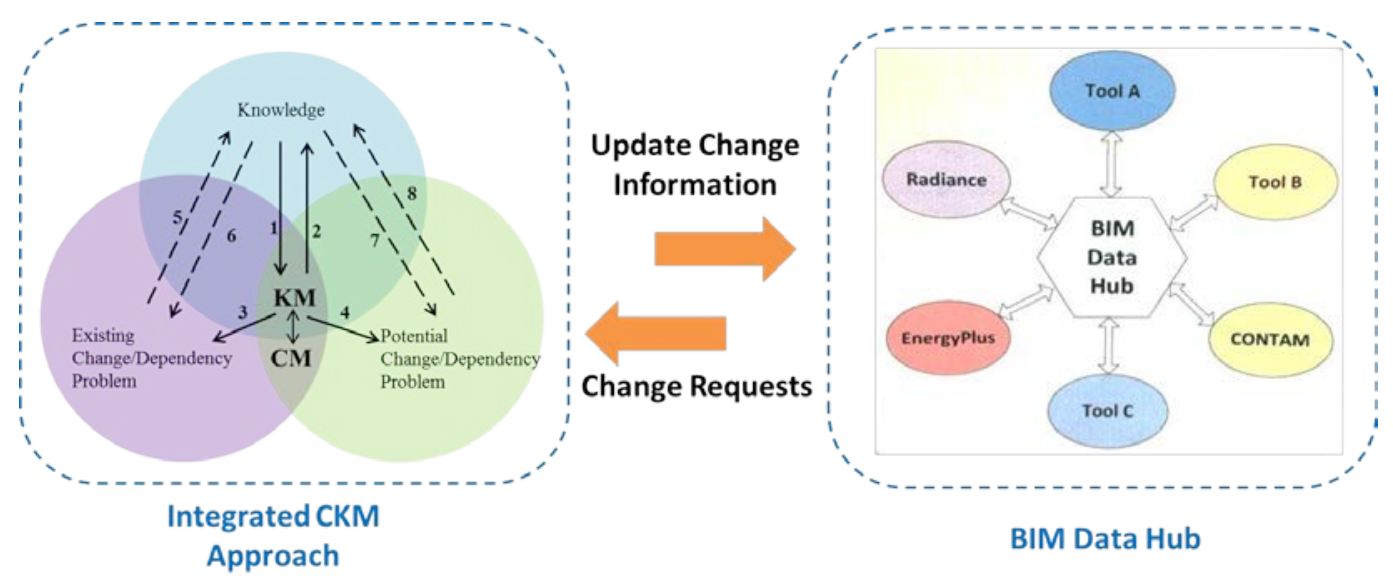

Figure 1: Adoption of the integrated CKM approach 
From the perspective of system development, the change and knowledge management system (CKMS), which was developed based on the integrated CKM approach, is enhanced to communicate with the BIM Data Hub using the JSON file format. As BIMserver can only accept IFC files, a new IFC file needs to be imported to replace the old content in order to update BIMserver. This function is enabled by an intermediate tool called 'Change Sync', which is capable of reading change information from the JSON file and updating the IFC file. In order to update the IFC file, it will identify the geometry information in the JSON file which has been approved for change. It will then retrieve the IFC file and match the geometry information in the JSON file to that of the IFC file, and update as necessary.

The system mechanism is shown in Figure 2. When the energy engineer comes up with a change request based on energy analysis, it will be put into CKMS for change approval, lessons learned capture, and knowledge management, etc. If it is approved, a JSON file that contains the change information will be generated by CKMS, and sent to Change Sync to generate a new version of IFC file as well as updating BIMserver. In this way, the change is efficiently managed and updated in IFC models.
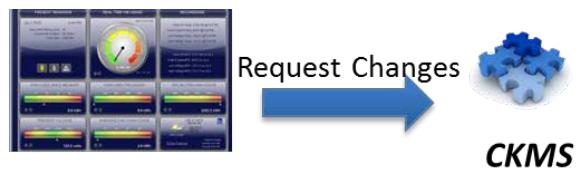

Notification $\rightarrow$ Change Approval Process

Energy Analysis

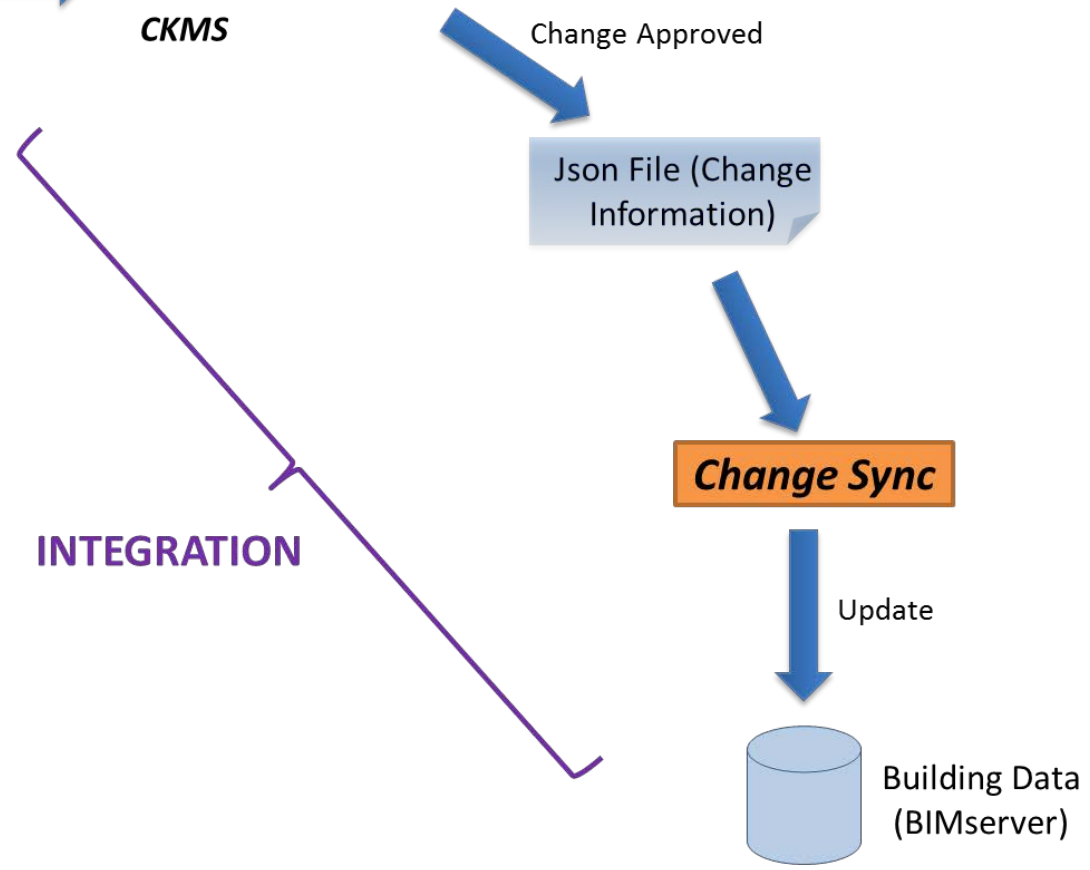

Figure 2: Framework to integrate CM with BIM

\section{USE CASE}

An airflow analysis use case was developed to demonstrate the framework. For better energy performance, the geometry information of a building is retrieved from the IFC 
model and converted into an OpenStudio file. The OpenStudio file will then be simulated in CONTAM by the mechanical engineer. In this process, the engineer may request a change of building partitions. This change request will be properly managed by the CKMS and updated in BIMserver in an automated workflow (as shown in Figure 3). Four main steps have been identified in this process:

(1) An IFC file is extracted from the model and put into BIMserver, where an OpenStudio file is generated by the query function for airflow analysis in CONTAM.

(2) To achieve energy efficient goals, a change request regarding the new partition design is proposed through CKMS by the mechanical engineer. The new request will then go through the approval process, which will include checking of dependencies, impact analysis, etc. The outcome of the approval will be communicated as change notifications (i.e., if approved) to the teams that need to know about the change.

(3) Once the request is approved, a JSON file containing the information on the partition change will be generated by the CKMS and sent to Change Sync tool. The tool will identify the change request and generate a new IFC file by updating geometry information in the old one.

(4) The new IFC file will be imported into BIMserver to replace the old content. Change Sync will also trigger the query function in BIM Data Hub to generate a new OSM file for energy analysis.

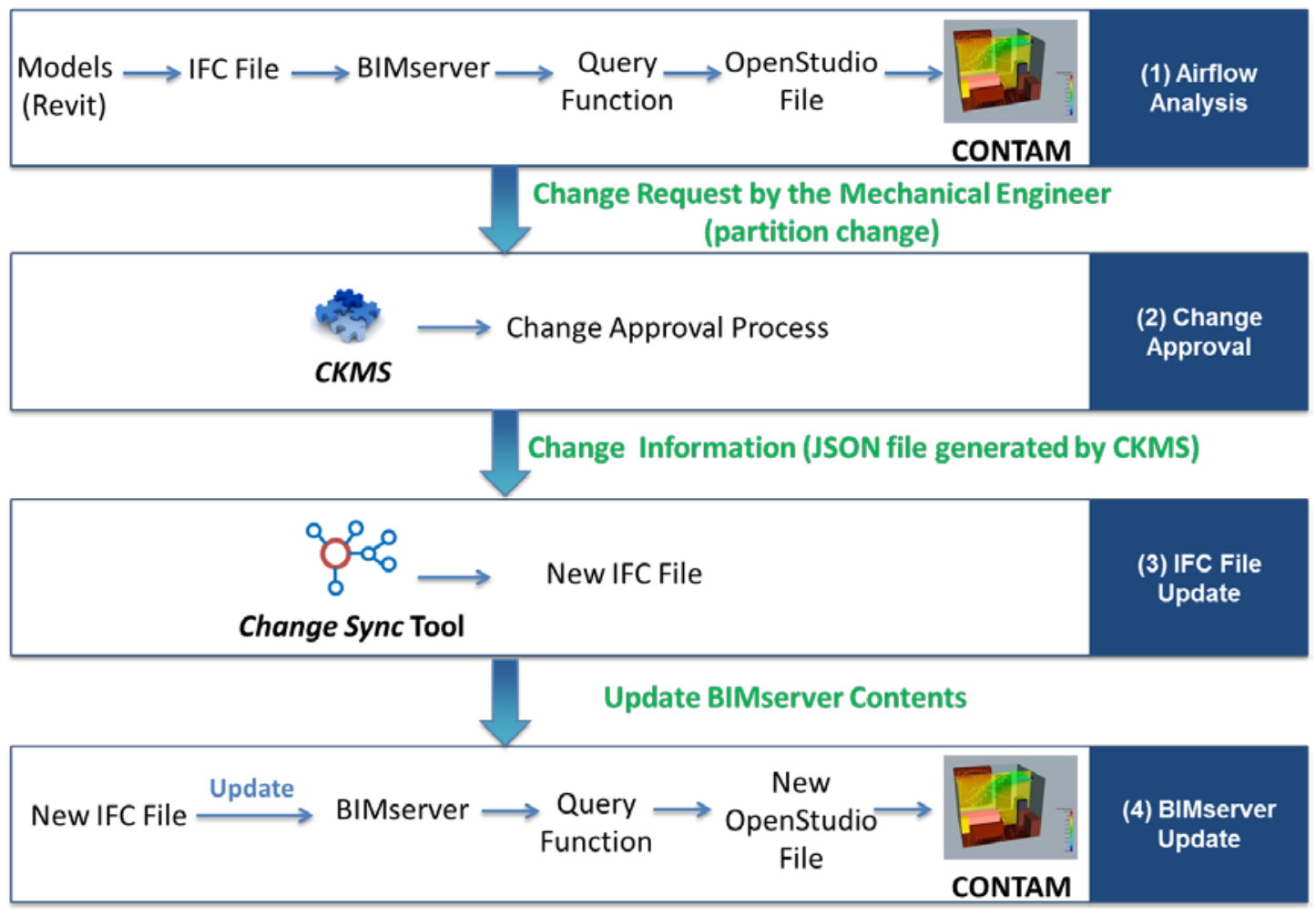

Figure 3: Use case workflow 


\section{ANALYSIS AND DISCUSSION}

Langroodi and Staub-French (2012) explored the possibility of managing changes with BIM, and evaluated the feasibility of some commercial BIM tools to check and manage changes. Their work mainly focused on the identification of changes in BIM models by checking and comparing different versions and the documentation of changes into certain categorizations. It is a post-change procedure and is only able to manage changes that have already been made on models. It does not support change approval processes or other change management activities that are not directly made on models.

In contrast, the integrated framework described in this paper enables the information update process on a BIM model, and facilitates the information flow and exchange for energy efficient design. It provides a workflow to capture and manage change information, and demonstrates an automated mechanism to update changes on BIM models. All change history is stored in the repository in CKMS so that team members can readily access it for reference. Instead of the post-change procedure, the framework shows how to integrate change management with BIM activities throughout the lifecycle of a project. A new change request is managed from the very beginning through the approval and implementation processes, and finally, the updating of BIM models. This provides an integrative and collaborative environment for energy efficient design. The adoption of the integrated CKM approach in this framework establishes a systematic approach to the management of changes, and introduces a comprehensive method to managing and propagating changes (rather than only managing model-related changes). Moreover, the framework can also be used to capture potential knowledge (lessons learned) from changes and help build a project knowledge repository. It will equip teams to be more proactive with change management issues.

As for the limitations, the system implementation is currently in progress and is not presented in this paper. It is proposed to develop the intermediate tool, Change Sync, as a separate module from the query function in the BIM Data Hub. The tool will act as a medium for communication between the CKMS and the BIM Data Hub, and it will be able to generate new IFC files and update BIM models based on change information. It is also intended to adopt version control techniques in Change Sync. All versions of IFC models will be stored for the purpose of tracking historical information and auditability. In a situation where it is required to revert to a previous unchanged version, the system will be able to retrieve the requested version for use.

\section{CONCLUSIONS}

Change management is very important in the achievement of energy efficiency goals in retrofit projects. BIM is being implemented widely in the construction industry, and information held on BIM models changes constantly during the lifecycle of buildings. These changes are often not managed adequately and updates are not reflected automatically on all aspects of the models. The process to request and approve changes is also not transparent thoroughly, thus inhibiting the capability to audit the process. This results in laborious efforts to update the models as well as 
consuming a lot of time in an error-prone way. Therefore, it is extremely vital to develop an efficient mechanism to support the management of changes within BIM. A framework was developed in this research to integrate CM with BIM. It contributes to an automated model updating workflow, a better method for information collection, and a more effective coordination process among team members. This provides a new integrative and efficient way to manage project changes, and it is able to help reduce the negative impacts of changes on energy efficiency goals. The framework can also be adapted and implemented in other types of construction projects in addition to energy efficient retrofits. Future work will focus on the evaluation of the framework within the construction industry and the development of the software prototype to demonstrate the process.

\section{ACKNOWLEDGMENTS}

We recognize the important contributions of the faculty and graduate students at the Department of Architectural Engineering, and College of Information Sciences and Technology, the Pennsylvania State University. This research is supported in part by the U.S. Department of Energy (DOE) under the EEB Hub Project. The opinions and views expressed here are those of the authors only.

\section{REFERENCES}

BiMserver.org. < http://bimserver.org/> (January 2014).

Eastman, C., Teicholz, P., Sacks, R., and Liston, K. (2011) BIM handbook: a guide to Building Information Modeling for owners, managers, designers, engineers and contractors, Wiley \& Sons, Inc., Hoboken, New Jersey.

Hao, Q., Shen, W., and Neelamkavil, J. (2008). "Managing changes in construction." NRC Report -RR-258. <http://www.nrc-cnrc.gc.ca/obj/irc/doc/pubs/rr/rr258/ rr258.pdf> (March 2012).

Jallow A.K., Liu F., Lee S., Anumba C.J., and Messner J.I. (2013). "An approach to integrate change and knowledge management in construction workflows." 30th International Conference on Applications of IT in the AEC Industry. Beijing, China. Oct. 2013.

Jiang, Y., Ming, J., Wu, D., Yen, J., Mitra, P., Messner, J., and Leicht, R. (2012). "BIM server requirements to support the energy efficient building lifecycle." Proceedings of the 2012 ASCE International Conference on Computing in Civil Engineering (ASCE 2012), Special Session on BIM Computer Science Fundamentals, Clearwater Beach, FL, USA, June 17-20, 2012.

LANGROODI, B., and STAUB-FRENCH, S. (2012). "Change management with Building Information Models: a case study.” Construction Research Congress, 2012. 1182-1191.

Liu F., Jallow A.K., Anumba C.J., and Wu D. (2013a). "Building Knowledge Modeling: integrating knowledge in BIM." 30th International Conference on Applications of IT in the AEC Industry. Beijing, China. Oct. 2013. 
Liu, F., Jallow, A., Anumba, C., and Messner, J. (2013b). "Integration of change and knowledge management processes in energy efficient retrofit projects." Proceedings of 2013 ASCE International Workshop on Computing in Civil Engineering, Los Angeles, CA, USA.

Love, P.E.D., MANDAL, P., and Li, H. (1999). "Determining the causal structure of rework influences in construction." Construction Management and Economics, 17, 505-517.

Motawa, I.A., Anumba, C.J., Lee, S., and Peña-Mora, F. (2007). "An integrated system for change management in construction." Automation in Construction, 16, 368-377.

Sanvido, V. E., and Riggs, L. S. (1993). "Managing successful retrofit projects.” Cost Engineering, 35, 25-31.

Sun, M., Sexton, M., Aouad, G., Fleming, A., Senaratne, S., Anumba, C., Chung, P., El-Hamalawi, A., Motawa, I., and Yeoh, M. L. (2004). "Managing changes in construction projects." EPSRC Industrial Report, University of the West of England: MCD Research Project. <http://www.bne.uwe.ac.uk/cprc/ publications/mcd.pdf $>$ (March 2012).

Taylor, J., and Bernstein, P. (2009). "Paradigm trajectories of Building Information Modeling practice in project networks." Journal of Management in Engineering, 25, 69-76.

Voropajev, V. (1998). "Change management-A key integrative function of PM in transition economies." International Journal of Project Management, 16, 1519.

Yu, N., Jiang, Y., Luo, L., Lee, S., Jallow, A., Wu, D., Messner, J., Leicht, R., and Yen, J. (2013). "Integrating BIMserver and OpenStudio for energy efficient building." Proceedings of 2013 ASCE International Workshop on Computing in Civil Engineering, Los Angeles, CA, USA. 